\title{
OmniSearch: a semantic search system based on the Ontology for MlcroRNA Target (OMIT) for microRNA-target gene interaction data
}

\author{
Jingshan Huang ${ }^{1 *}$, Fernando Gutierrez ${ }^{2}$, Harrison J. Strachan ${ }^{1}$, Dejing Dou ${ }^{2}$, Weili Huang ${ }^{3}$, Barry Smith ${ }^{4}$, \\ Judith A. Blake ${ }^{5}$, Karen Eilbeck ${ }^{6}$, Darren A. Natale ${ }^{7}$, Yu Lin ${ }^{8}$, Bin Wu ${ }^{9}$, Nisansa de Silva², Xiaowei Wang ${ }^{10}$, \\ Zixing Liu ${ }^{11}$, Glen M. Borchert ${ }^{12}$, Ming Tan ${ }^{11}$ and Alan Ruttenberg ${ }^{13}$
}

\begin{abstract}
As a special class of non-coding RNAs (ncRNAs), microRNAs (miRNAs) perform important roles in numerous biological and pathological processes. The realization of miRNA functions depends largely on how miRNAs regulate specific target genes. It is therefore critical to identify, analyze, and cross-reference miRNA-target interactions to better explore and delineate miRNA functions. Semantic technologies can help in this regard. We previously developed a miRNA domain-specific application ontology, Ontology for MlcroRNA Target (OMIT), whose goal was to serve as a foundation for semantic annotation, data integration, and semantic search in the miRNA field. In this paper we describe our continuing effort to develop the OMIT, and demonstrate its use within a semantic search system, OmniSearch, designed to facilitate knowledge capture of miRNA-target interaction data. Important changes in the current version OMIT are summarized as: (1) following a modularized ontology design (with 2559 terms imported from the NCRO ontology); (2) encoding all 1884 human miRNAs (vs. 300 in previous versions); and (3) setting up a GitHub project site along with an issue tracker for more effective community collaboration on the ontology development. The OMIT ontology is free and open to all users, accessible at: http://purl.obolibrary.org/obo/omit.owl. The OmniSearch system is also free and open to all users, accessible at: http://omnisearch.soc.southalabama.edu/index.php/Software.
\end{abstract}

Keywords: microRNA, Non-coding RNA, Target gene, Biomedical ontology, Ontology development, Data annotation, Data integration, Semantic search, SPARQL query

\section{Introduction}

microRNAs (miRNAs) are a type of non-coding RNA (ncRNA) with important biological, biomedical, and clinical impact. Prior research $[1,2]$ indicates that miRNAs perform significant roles in both biological and pathological processes, thus affecting the control and regulation of various human diseases. miRNAs realize critical functions via binding to their respective target genes. The ability to identify and analyze miRNA-target interactions in an

\footnotetext{
*Correspondence: huang@southalabama.edu

1 School of Computing, University of South Alabama, Mobile, Alabama 36688-0002, USA

Full list of author information is available at the end of the article
}

effective manner is thus a key step in the understanding and delineation of miRNA functions.

The conventional method by which the users of data (e.g., biologists, bioinformaticians, and clinical investigators) determine miRNA functions involves:

- Searching for biologically validated miRNA targets, for example, by querying the PubMed database [3]; and

- Finding additional potential miRNA targets, for example, by initiating inquiries on various prediction databases or websites such as miRDB [4], TargetScan [5], and miRanda [6]. 
Unfortunately, both steps currently require significant manual effort because the relevant data sources are both syntactically and semantically heterogeneous - that is, the meaning of seemingly similar data from different sources may be quite different and thus open to misinterpretation. It is therefore challenging for users to identify and establish possible links among original data sources. As a result, conventional miRNA knowledge discovery and acquisition methodologies are timeconsuming, labor-intensive, error-prone, and sensitive to limitations in the prior knowledge of different end users. These barriers are exacerbated by the need to obtain additional information for each and every miRNA target (whether validated or putative) using existing data sources and analysis tools, including but not limited to: the DAVID Bioinformatics Resources (DAVID) [7], NCBI Gene [8], the Medical Subject Headings (MeSH) Database [9], the HUGO Gene Nomenclature Committee (HGNC) Database [10], and NCBI Nucleotide [11].

Emerging semantic technologies can help in addressing the aforementioned challenges. The core of current semantic technologies include specifications such as the resource description framework (RDF), RDF Schema (RDFS), and Web Ontology Language (OWL), all of which are intended to provide a formal description of classes of entities of different types and of the relations between them in such a way as to enable automatic reasoning (inference). Semantic technologies can be applied to miRNA knowledge acquisition by transforming data obtained from heterogeneous miRNA-related databases into a common framework by utilizing a single format (such as RDF) and aligning the data through use of annotations from common, formally defined ontologies. By means of this transformation we can use the SPARQL Protocol (SPARQL) [11] to query the enhanced data automatically.

In previous research [12-17], we investigated the construction of an application ontology for the miRNA field, named Ontology for MIcroRNA Target (OMIT), the first ontology to formally encode miRNA domain knowledge. By providing a standardized metadata model to establish miRNA data connections among heterogeneous sources, the OMIT is able to fill two gaps: the lack of common data elements and the lack of data exchange standards for miRNA research, especially with regard to miRNA-target interactions.

We describe two major scientific contributions in this paper: (1) recent improvements to the OMIT ontology and (2) a semantic search system, which is built upon the ontology and enables the capture of miRNA-target interaction data in a way leading to more effective miRNA knowledge acquisition.

The remainder of this paper is organized as follows. "Related work" Section summarizes state-of-the-art research in biomedical ontologies and semantic search, respectively. "OMIT reconstruction" Section reports our efforts on reconstructing the OMIT ontology. "OmniSearch: an OMIT-based semantic search system" Section describes technical details of OmniSearch, an OMIT-based semantic search system. "Results and discussion" Section reports our experimental results. Finally, "Conclusions" Section summarizes the major points and presents ideas for future research.

\section{Related work}

\section{Related work in biomedical ontologies}

The use of ontologies to describe, define, and integrate biological entities has long been embraced by the biological, biomedical, and clinical research communities. Here we briefly describe some representative bio-ontologies included in both the Open Biological and Biomedical Ontologies (OBO) Library [18] and the National Center for Biomedical Ontology (NCBO) BioPortal [19] that are pertinent to the development of this project.

The Gene Ontology (GO) [20] is by far the most successful and widely used ontology for biological data description. It consists of three independent subontologies: biological processes, molecular functions, and cellular components, which describe these aspects of gene products: both protein and RNA. The GO has been widely utilized to annotate gene products of model organisms. By the time of writing this paper, there were GO annotations for 36 organisms including Homo sapiens available for download.

The Sequence Ontology (SO) [21] is an ontology to capture genomic features and the relationships that obtain between them. This ontology contains the features necessary to annotate a genome with structural features such as gene models and also the terms necessary for the annotation of genomic variants. SO terms define the kinds of and parts of ncRNA features, and these terms are used to identify these features and their location in genomic sequence.

The PRotein Ontology (PRO) [22] is a comprehensive description of the forms of protein, including isoforms, modifications, and the relationships between them. Proteins are functional entities in many processes eventually impacted by the regulatory effect of ncRNAs (e.g., miRNA bindings). The PRO provides an ontological representation of proteins with a particular focus on human proteins and disease-related variants thereof.

The RNA Ontology (RNAO) [23] is a candidate OBO foundry reference ontology to catalogue the molecular entities composing primary, secondary, and tertiary components of RNA. The goal of this project is to enable integration and computation over diverse RNA datasets. 


\section{Related work in semantic search}

Semantic search is a research field that intends to improve the access to contents by considering the semantics behind the search process [24]. In other words, semantic search goes beyond conventional, keyword-based search by considering the contextual meaning of words, the intent of the user, and the nature of the search space. In general, semantic search requires the use of structured knowledge, such as ontologies, in the modeling and interpretation of queries. Ontologies can help improve the search by query expansion. One main idea in many semantic search systems (e.g., [25-29]) is, the original set of query keywords can be expanded by drawing on synonyms and other relationships (e.g., subclass and parthood) that are not part of the query. For example, in the work by Chauhan et al. [29], the original query was first expanded by considering synonyms, then terms with high semantic similarity were chosen from the ontology to be integrated to the search query, and the semantic similarity used for the query expansion was computed by the distance among concepts in the ontology, the position in the hierarchy, and the number of upper classes.

Another way to implement semantic search is to use ontologies to translate keyword-based search into formal semantic queries. For example, Tran et al. [24] used a set of models (mental, user, system, and query) to capture information, such as thought entities, language primitives, knowledge representation (KR) primitives, and query elements. These models were then combined with a set of assumptions to redefine original queries, filling the gap between terms with structural information from an ontology. That is, each term within the query was considered a property of another term.

\section{OMIT reconstruction}

\section{Modularized ontology design}

The OMIT ontology consists of the following modules:

- omit.owl - Defines all OMIT-specific terms and relations, for example, prediction_from_miRDB and gene_context_score_in_TargetScan.

- bfo.owl - Imports upper-level terms from the Basic Formal Ontology (BFO) [30], for example, generically dependent continuant and material entity.

- ro-imports.owl - Imports common relations (shared across different ontologies) from the Relation Ontology (RO) [31], for example, has participant and regulates.

- ncro.owl - Imports ncRNA-related terms and relations from the Non-coding RNA Ontology (NCRO) [32], for example, miRNA_target_gene and miRNA_gene_family.
- go-imports.owl - Imports gene product terms from the GO, for example, RNA binding and regulation of biological process.

- so-imports.owl - Imports sequence structural feature terms from the $\mathrm{SO}$, for example, biological_region and insertion_site.

- obi-imports.owl - Imports life-science and clinical investigation terms from the Ontology for Biomedical Investigations (OBI) [33], for example, cultured cell population and organism.

- chebi-imports.owl - Imports molecular entity (especially small chemical compounds) terms from the Chemical Entities of Biological Interest Ontology (ChEBI) [34], for example, ribonucleic acid and ribosomal RNA.

- iao-imports.owl - Imports information entity terms from the Information Artifact Ontology (IAO) [35], for example, information content entity.

- clo-imports.owl - Imports cell line-relevant terms from the Cell Line Ontology (CLO) [36], for example, cell line.

- pr-imports.owl - Imports protein-related entity terms from the PRO, for example, amino acid chain and protein.

- uberon-imports.owl - Imports cross-species anatomy terms from the Uberon multi-species anatomy ontology (UBERON) [37], for example, anatomical structure and organ.

- doid-imports.owl - Imports disease terms from the Human Disease Ontology (DOID) [38], for example, disease of cellular proliferation and cancer.

Note that:

(1) Orthogonality among different ontologies is one of the important practices proposed by the $\mathrm{OBO}$ Foundry Initiative, and has been widely accepted in the bio-ontology community. As a result, to achieve better orthogonality, it is a common practice to reuse contents defined in relevant, existing ontologies.

(2) The OMIT ontology directly imported the NCRO ontology (a comprehensive ncRNA domain ontology), which in turn, directly imported other ontologies in the above list. Therefore, the OMIT ontology itself includes two OWL files: "omit.owl" and "ncro.owl." All other OWL files, "go-imports.owl" and "so-imports.owl" for example, are shown as "indirectly imported" in Protégé.

(3) Ontology concepts are referred to as "classes" in Protégé and "terms" in OBO Edit, respectively. Therefore, "classes" and "terms" are interchangeably used throughout the whole paper.

Table 1 lists a subset of important terms and relations imported into the OMIT. 
Table 1 A subset of imported terms and relations

\begin{tabular}{|c|c|c|}
\hline Imported term or relation & Source ontology & Original ID \\
\hline RO:part of & Relation Ontology & BFO_0000050 \\
\hline RO:participates in & Relation Ontology & RO_0000056 \\
\hline RO:has participant & Relation Ontology & RO_0000057 \\
\hline BFO:entity & Basic Formal Ontology & BFO_0000001 \\
\hline BFO:continuant & Basic Formal Ontology & BFO_0000002 \\
\hline BFO:independent continuant & Basic Formal Ontology & BFO_0000004 \\
\hline BFO:occurrent & Basic Formal Ontology & BFO_0000003 \\
\hline BFO:material entity & Basic Formal Ontology & BFO_0000040 \\
\hline CHEBI:molecular entity & Chemical Entities of Biological Interest Ontology & CHEBI_23367 \\
\hline CHEBl:ribonucleic acid & Chemical Entities of Biological Interest Ontology & CHEBI_23367 \\
\hline CHEBl:ribosomal RNA & Chemical Entities of Biological Interest Ontology & CHEBI_18111 \\
\hline CHEBI:small nuclear RNA & Chemical Entities of Biological Interest Ontology & CHEBI_74035 \\
\hline CHEBI:transfer RNA & Chemical Entities of Biological Interest Ontology & CHEBI_17843 \\
\hline NCRO:human_miRNA & Non-coding RNA Ontology & NCRO_0000810 \\
\hline NCRO:hsa-miR-125b-1-3p & Non-coding RNA Ontology & NCRO_0003283 \\
\hline NCRO:hsa-miR-125b-2-3p & Non-coding RNA Ontology & NCRO_0003284 \\
\hline NCRO:hsa-miR-125b-5p & Non-coding RNA Ontology & NCRO_0003282 \\
\hline NCRO:miRNA_target_gene & Non-coding RNA Ontology & NCRO_0000025 \\
\hline NCRO:miRNA_and_target_gene_binding & Non-coding RNA Ontology & NCRO_0000003 \\
\hline NCRO:protein_miRNA_promoter_binding & Non-coding RNA Ontology & NCRO_0000011 \\
\hline IAO:information content entity & Information Artifact Ontology & IAO_0000030 \\
\hline IAO:measurement datum & Information Artifact Ontology & IAO_0000109 \\
\hline
\end{tabular}

- The format for the left column (Imported Term or Relation) is PREFIX:human-readable label, for example, NCRO:miRNA_target_gene and RO:part of.

- The format for the right column (Original ID) is PREFIX_unique identifier, for example, NCRO_0000025 and BFO_0000001.

\section{Ontology core design}

The core design of the OMIT ontology is shown in Fig. 1. Compared with earlier versions, the current version contains many important new terms and relations, and some of which are listed in Tables 2 and 3, respectively.

- Both terms and relations are represented in the format of PREFIX:label in Fig. 1.

- For the purpose of better readability, labels rather than identifiers are used in Tables 2 and 3.

- Relations in Table 3 were either defined in or imported into the OMIT, which can be easily distinguished from each other by different prefixes used in the first column.

\section{OmniSearch: an OMIT-based semantic search system}

Based on the OMIT ontology, we developed a semantic search system: OmniSearch. First, the OmniSearch system will conduct semantic annotation on various sources that were originally heterogeneous in their semantics; following that, OMIT-annotated data will then be integrated into a unified and consistent data layer in RDF; and finally, complex semantic queries will be performed to provide meaningful results and clues to system end users (e.g., biologists, bioinformaticians, and clinical investigators).

\section{Data sources used}

Data sources used in the OmniSearch system include three miRNA target prediction databases (miRDB, TargetScan, and miRanda), as well as PubMed, NCBI Gene, GO, RNA Central, DAVID, HGNC, and $\mathrm{MeSH}$ term databases. These sources contain both structured data (database instances) and unstructured data (free text), and are semantically heterogeneous among each other.

\section{Software architecture}

The OmniSearch system consists of several software modules: semantic annotation, data integration, and semantic search.

Semantic data annotation is the process of tagging source files with predefined ontological metadata like names, entities, attributes, definitions, and descriptions. The annotation provides original data with extra metadata 


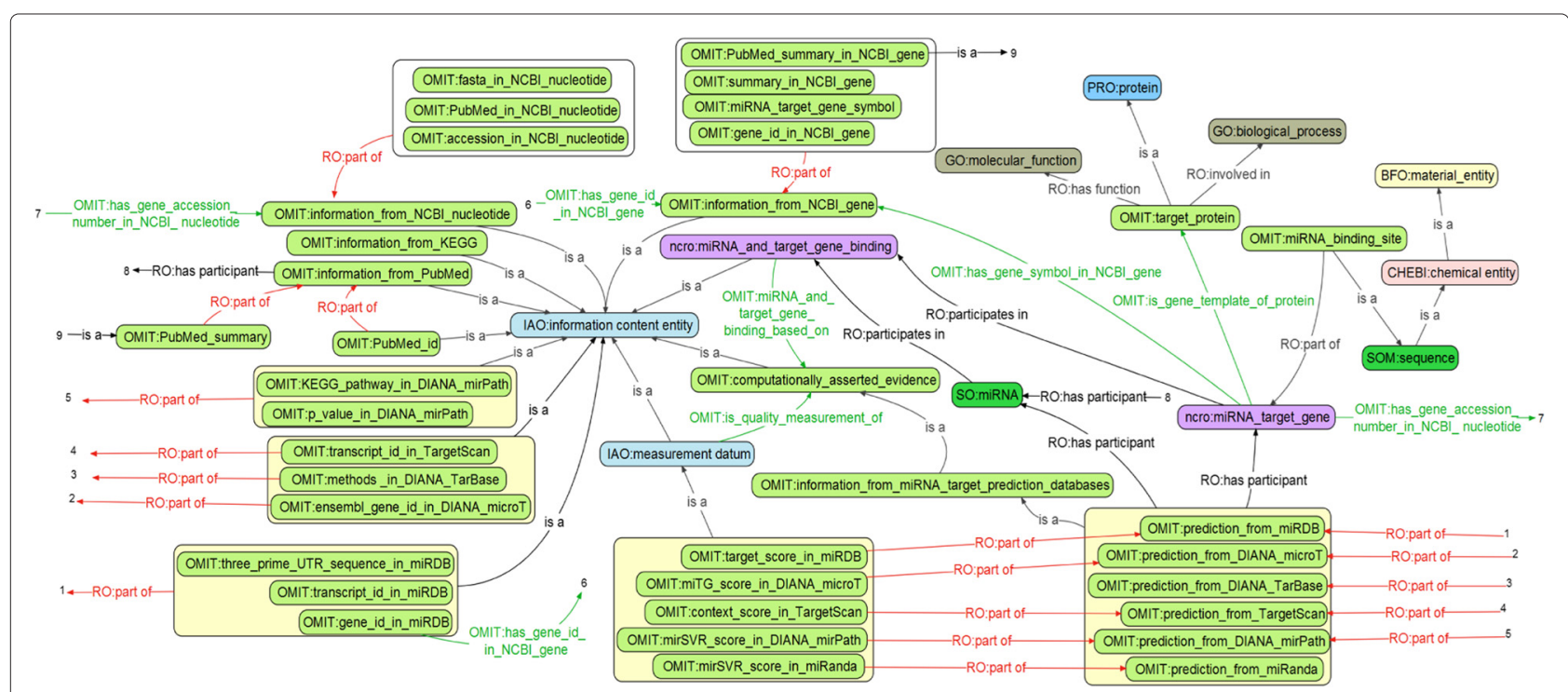

Fig. 1 The design of core terms and relations in the OMIT ontology (both terms and relations are represented in the format of PREFIX:label)

Table 2 A subset of new OMIT terms

\begin{tabular}{|c|c|c|}
\hline OMIT new term & Direct parent term & Human-readable explanation \\
\hline \multirow[t]{2}{*}{ computationally_asserted_evidence } & \multirow[t]{2}{*}{ IAO:Information content entity } & Evidence obtained from some \\
\hline & & computational methods. \\
\hline information_from_miRNA_ & \multirow[t]{2}{*}{ OMIT:computationally_asserted_evidence } & Records obtained from various \\
\hline target_prediction_database & & miRNA target prediction databases. \\
\hline \multirow[t]{2}{*}{ prediction_from_miRDB } & OMIT:information_from_miRNA_ & Records specifically obtained \\
\hline & target_prediction_database & from the miRDB database. \\
\hline \multirow[t]{2}{*}{ prediction_from_TargetScan } & OMIT:information_from_miRNA_ & Records specifically obtained \\
\hline & target_prediction_database & from the TargetScan database. \\
\hline \multirow[t]{2}{*}{ prediction_from_miRanda } & OMIT:information_from_miRNA_ & Records specifically obtained \\
\hline & target_prediction_database & from the miRanda database. \\
\hline \multirow[t]{3}{*}{ target_score_in_miRDB } & IAO:measurement datum & The score of some specific \\
\hline & & miRNA-target binding prediction \\
\hline & & from the miRDB database. \\
\hline \multirow[t]{3}{*}{ gene_context_score_in_TargetScan } & IAO:measurement datum & The context score of some specific \\
\hline & & miRNA-target binding prediction \\
\hline & & from the TargetScan database. \\
\hline \multirow[t]{3}{*}{ mirSVR_score_in_miRanda } & IAO:measurement datum & The mirSVR score of some specific \\
\hline & & miRNA-target binding prediction \\
\hline & & from the miRanda database. \\
\hline \multirow[t]{2}{*}{ information_from_NCBI_gene } & IAO:information content entity & Records obtained from NCBI Gene \\
\hline & & according to gene IDs or gene symbols. \\
\hline \multirow[t]{2}{*}{ information_from_NCBI_nucleotide } & IAO:information content entity & Records obtained from NCBI Nucleotide \\
\hline & & according to GenBank Accession numbers. \\
\hline \multirow[t]{2}{*}{ information_from_PubMed } & IAO:information content entity & Records obtained from the PubMed \\
\hline & & database according to PMIDs. \\
\hline
\end{tabular}


Table 3 A subset of new OMIT relations

\begin{tabular}{|c|c|c|c|}
\hline New relation & Domain & Range & Human-readable explanation \\
\hline $\begin{array}{l}\text { OMIT:miRNA_target_ } \\
\text { assumption_ } \\
\text { based_on }\end{array}$ & $\begin{array}{l}\text { NCRO:miRNA_and_ } \\
\text { target_gene_binding }\end{array}$ & $\begin{array}{l}\text { OMIT:computationally_ } \\
\text { asserted_evidence }\end{array}$ & $\begin{array}{l}\text { Specific miRNA-target binding } \\
\text { prediction is based on some } \\
\text { computationally asserted evidence }\end{array}$ \\
\hline $\begin{array}{l}\text { OMIT:is_quality_ } \\
\text { measurement_of }\end{array}$ & IAO:measurement datum & $\begin{array}{l}\text { OMIT:computationally_ } \\
\text { asserted_evidence }\end{array}$ & $\begin{array}{l}\text { A piece of measurement datum } \\
\text { (e.g., the target score in miRDB) } \\
\text { is a quality measurement of } \\
\text { computationally asserted evidence }\end{array}$ \\
\hline $\begin{array}{l}\text { OMIT:is_gene_ } \\
\text { template_of_protein }\end{array}$ & NCRO:miRNA_target_gene & OMIT:target_protein & $\begin{array}{l}\text { A miRNA target gene } \\
\text { serves as a template } \\
\text { of relevant protein. }\end{array}$ \\
\hline RO:has participant & OMIT:prediction_from_miRDB & SO:miRNA & $\begin{array}{l}\text { Each miRNA-target binding } \\
\text { prediction record has one } \\
\text { miRNA as a participant. }\end{array}$ \\
\hline RO:has participant & OMIT:prediction_from_miRDB & NCRO:miRNA_target_gene & $\begin{array}{l}\text { Each miRNA-target binding } \\
\text { prediction record has one } \\
\text { target as a participant. }\end{array}$ \\
\hline RO:part of & OMIT:target_score_in_miRDB & OMIT:prediction_from_miRDB & $\begin{array}{l}\text { Each miRNA-target binding } \\
\text { prediction record from } \\
\text { miRDB contains one score. } \\
\text { Each record from NCBI }\end{array}$ \\
\hline RO:part of & OMIT:PubMed_summary_ & $\begin{array}{l}\text { OMIT:information_from_NCBI_gene } \\
\text { in_NCBI_gene }\end{array}$ & $\begin{array}{l}\text { Gene contains one or } \\
\text { more PubMed summaries. }\end{array}$ \\
\hline
\end{tabular}

information formally defined in the OMIT ontology. The output of semantic data annotation is a collection of RDF triples (from both free text and database instances). These triples will be accumulated into a centralized RDF repository: OmniStore.

We used Python scripts to conduct automated semantic annotation and data integration. As an example, Fig. 2 shows the flowchart of our programs to annotate miRDB data. We explain below the detailed steps. One miRDB file, the "miRNA data" file, contains two columns consisting of miRNA names and their associated internationalized resource identifiers (IRIs). Another miRDB file, the "gene data" file, contains four columns consisting of miRNA names, gene IDs, gene symbols, and target scores.

- Step One: As each miRNA name and its associated IRI were read in from the miRNA data file, they were placed into a dictionary where the miRNA name is the key and the IRI is the value.

- Step Two: All lines were read in from the gene data file, and each line was converted into a total of four RDF triples. (1) The first triple was generated to represent a newly created instance of the
prediction_from_miRDB class, namely, instance_i, and a new OMIT IRI was assigned to instance_i. (2) Next, the miRNA name read from the same line was used to retrieve its corresponding IRI from the dictionary (generated in Step One). The second triple then connected this retrieved IRI with instance_i. (3) Two more triples were generated to connect instance_ $i$ with the corresponding gene ID and target score read in from the same line, respectively.

- Step Three: Finally, all generated RDF triples were written into a Turtle file.

Note that:

(1) "Semantic annotation and data integration" Section exhibits some example triples resulted from the above-mentioned annotation process.

(2) Mappings between database schemas and ontological entities were defined in the OMIT ontology and can be reused or modified in the future, when needed.

(3) Due to our automated annotation and integration techniques, only minimum effort will be required to integrate a new resource in the future. 


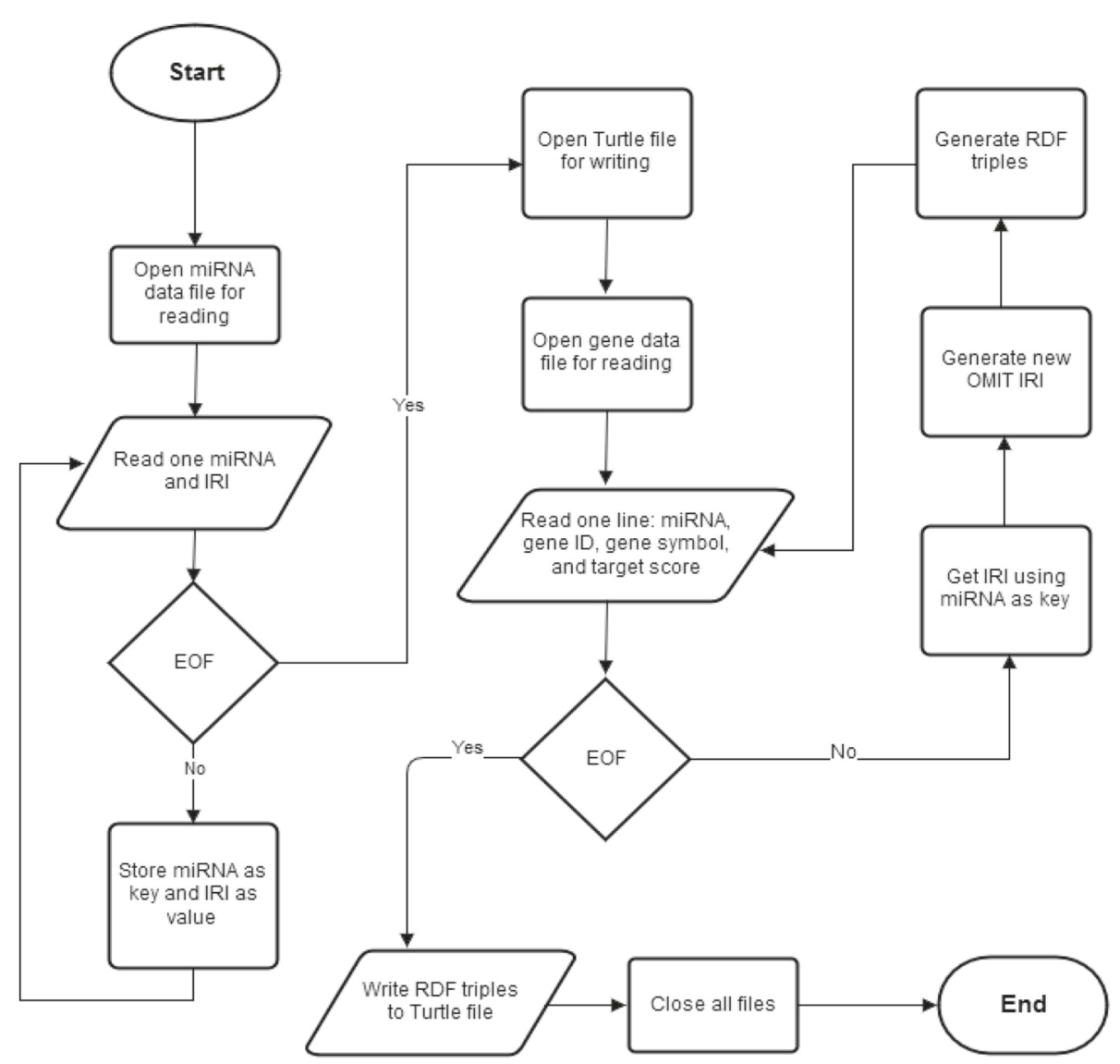

Fig. 2 Semantic annotation and data integration flowchart in the OmniSearch system

Because all semantic tags are to be generated from the global metadata model defined in the OMIT ontology, the RDF triple repository will provide a unified view over original data sources at semantic level. Consequently, complex semantic queries will be enabled. To implement semantic search, we made use of Apache HTTP server [39], PHP: Hypertext Preprocessor (PHP) server [40], and Apache Jena Fuseki server [41]. The overall software architecture is demonstrated in Fig. 3, with the following working protocol:

- Query parameters are sent from the client's browser to the Apache server through Ajax requests.

- SPARQL queries are dynamically generated by the Apache server using these query parameters, which are then sent to the Apache Jena Fuseki server.

- JSON objects, containing the requested information, are retrieved from the RDF triple store (installed on the Apache Jena Fuseki server) after running the dynamically generated SPARQL queries.

- These JSON objects are returned to the Apache server, which are used to generate either (1) a list of
miRNAs and/or MeSH terms or (2) the HTML Markup for the search result table.

- Finally, the Apache server sends the obtained data, or an error message if the search fails, back to the client's browser as a JSON object.

\section{User interface design}

The OmniSearch is a Web-based search system that is free and open to all users, accessible at: http://omnisearch. soc.southalabama.edu/index.php/Software. As shown in Fig. 4, the main components of the graphic user interface (GUI) are: two search criteria boxes, a search result table, a pagination control, a set of result viewing filters, a result download tool, and DAVID analysis functionality. More discussion on our friendly user interface design can be found in "Search results and discussion" Section.

\section{Results and discussion}

\section{The significantly refactored OMIT ontology}

The updated version of the OMIT ontology contains a total of 3169 terms and 46 relations (besides a total of 5515 is_a relations). Note that out of 46 relations 


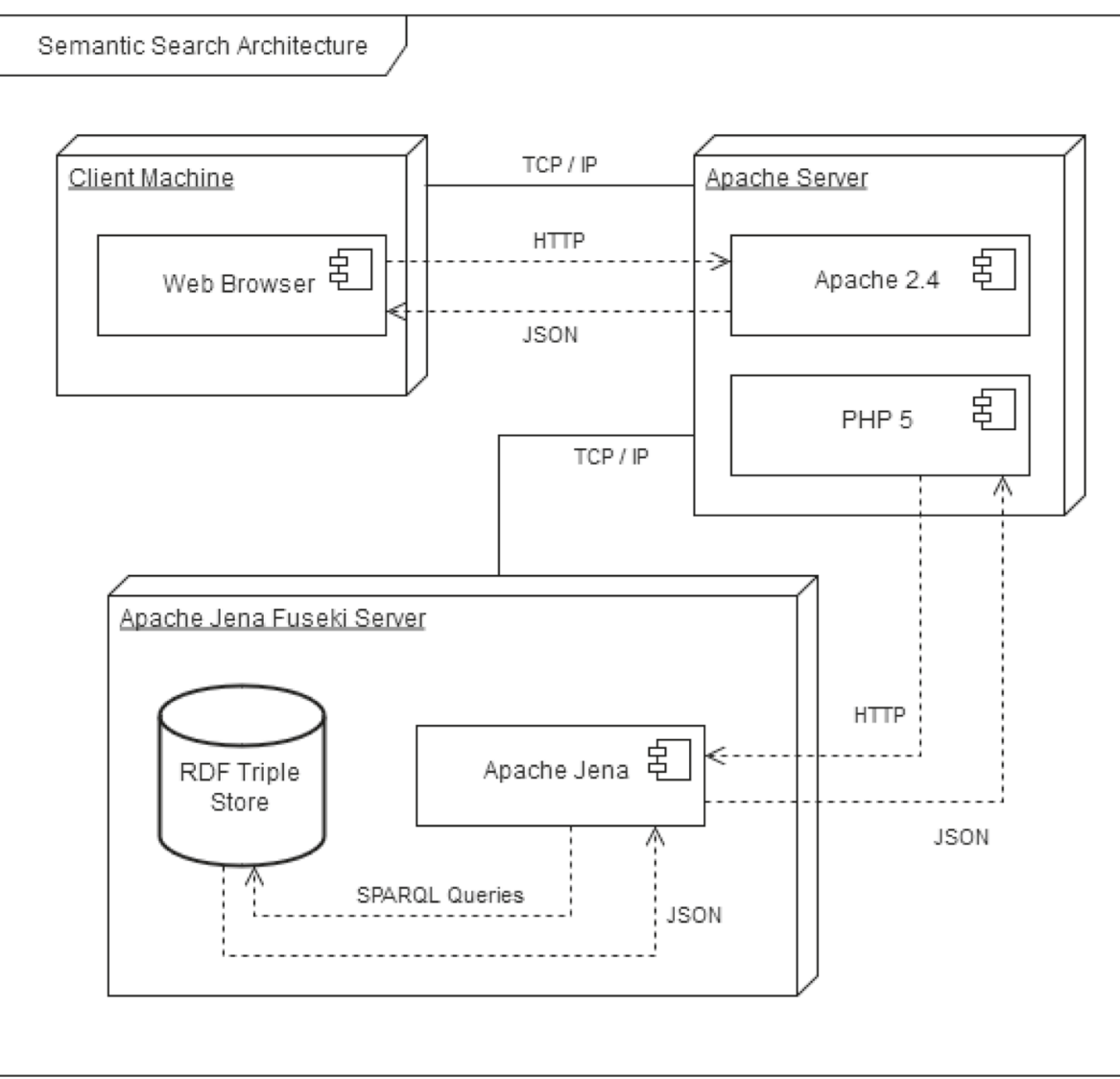

Fig. 3 Semantic search architecture in the OmniSearch system

mentioned here, there are 5 data properties, and the rest are object properties. Also note that these terms and relations include both OMIT-specific ones and those imported ones ${ }^{1}$.

Compared with the previous versions [12-17], important changes in the current version OMIT ontology are summarized as follows.

- As discussed earlier in "Modularized ontology design" Section, we have followed a modularized ontology design in this new version, which will significantly further facilitate the ontology maintenance and update. In particular, a total of 2559 terms in the updated OMIT have been imported from the NCRO ontology [32]. Because the NCRO is a comprehensive domain ontology in the ncRNA field, following the NCRO hierarchy will enhance the interoperability between the OMIT and future ontologies to be developed in other ncRNA sub-domains.

- In the previous versions of OMIT, around 300 human miRNAs were included. In the current version, all 1884 miRNAs appearing in humans have been encoded, along with the information about the gene family group of each and every miRNA. According to miRBase [42], there are a total of 320 different gene family groups. This information can be highly valuable because the fact that two or more miRNAs of interest indeed belong to the same gene family group can provide biologists, bioinformaticians, and clinical investigators with critical clues in constructing new hypothesis.

- In our previous investigations, we established a dedicated project website [43], as well as entries in both the OBO Library [44] and the NCBO BioPortal [45]. To further disseminate the ontology, and, to gather feedback from community in a more effective manner, we have recently created $\boldsymbol{a}$ GitHub project site (https://github.com/OmniSearch/omit) for this new version OMIT ontology. We have also established $\boldsymbol{a}$ tracker [46] for an enhanced mechanism in handling the discussion among groups to further improve the ontology. New concepts, definitions, and their locations in the OMIT can now be proposed, debated, and approved (or rejected) by an open group of individuals through this tracker. 


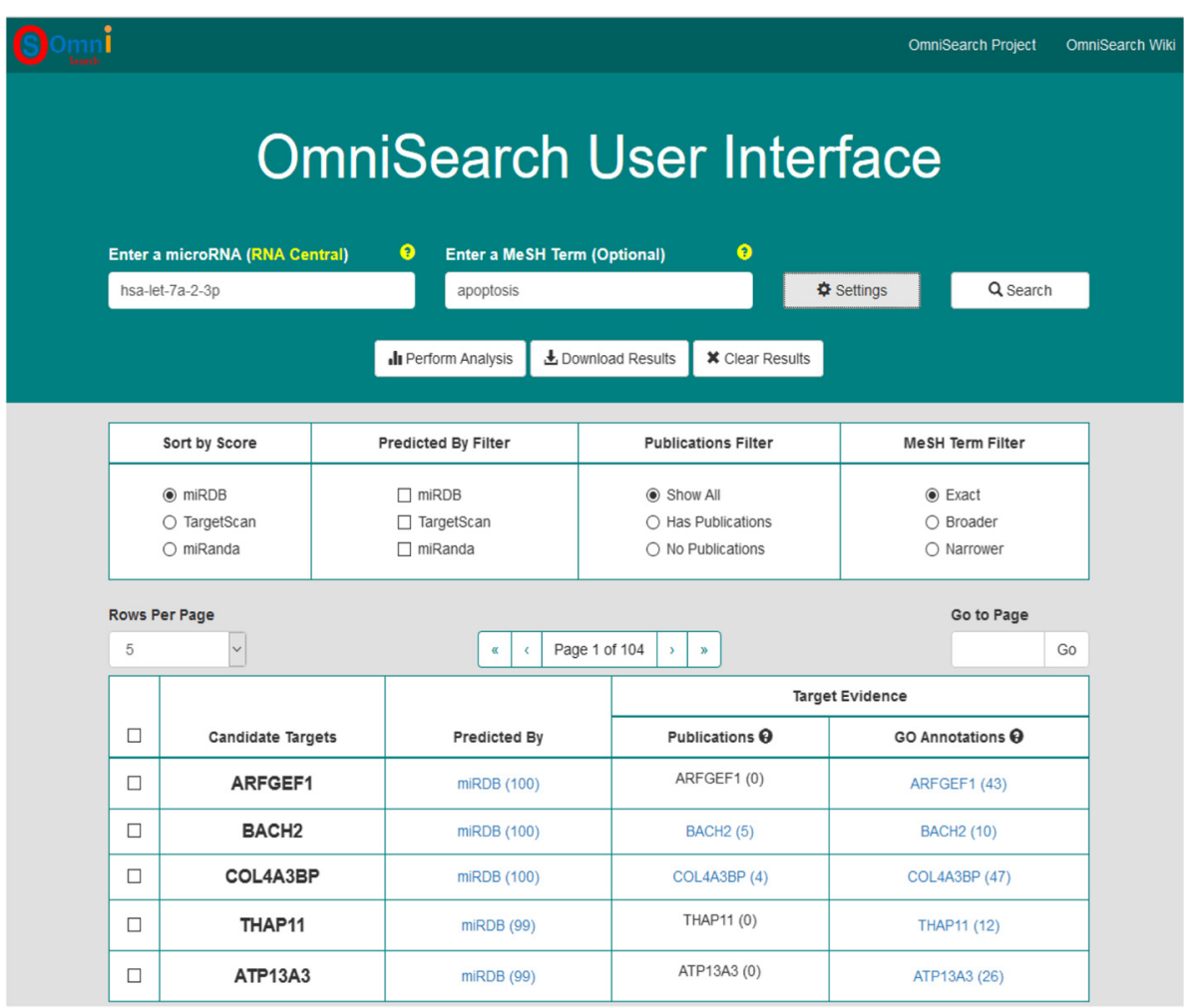

Fig. 4 GUI design in the OmniSearch system

\section{Semantic annotation and data integration Experimental setup}

The OmniStore RDF repository is housed on a server with the following configuration: Intel(R) Core(TM) i7-3632 QM CPU @ 2.80 GHz 2.80 GHz; 32.00 GB memory; and Windows Server 8 Operating System.

\section{Semantic annotation and data integration results}

OmniStore contains a total of 6,136,514 RDF triples, and the file size of OmniStore is $369 \mathrm{MB}$. All triples are represented in RDF 1.1 Turtle: Terse RDF Triple Language format [47], for example:

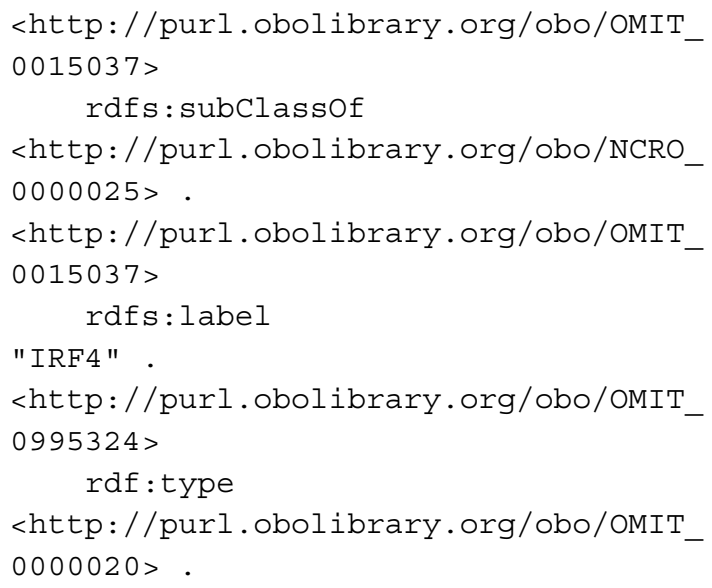

<http://purl.obolibrary.org/obo/oMIT_ $0995324>$

<http://purl.obolibrary.org/obo/RO_ $0000057>$

<http://purl.obolibrary.org/obo/OMIT_ $0015037>$.

<http://purl.obolibrary.org/obo/OMIT_ $0995324>$

<http://purl.obolibrary.org/obo/RO $0000057>$

<http://purl.obolibrary.org/obo/OMIT_ $0050688>$.

<http://purl.obolibrary.org/obo/OMIT_ $0995324>$

<http://purl.obolibrary.org/obo/OMIT_ $0000108>$

100 .

The semantics of the above six example triples is: IRF4 (OMIT_0015037) is a subclass of the miRNA_target_gene class (NCRO_0000025); one miRDB database record (OMIT_0995324), which is an instance of the prediction_from_miRDB class (OMIT_0000020), indicates that IRF4 is a predicted target of the miRNA hsamiR-125b-5p (OMIT_0050688); and the prediction score (OMIT_0000108) is 100. 


\section{Semantic search}

We use one example in this section to demonstrate in detail how the OmniSearch system assists in end users' knowledge acquisition.

\section{Experimental setup}

Semantic search was conducted on a personal computer (PC) with the following configuration: Intel(R) Core(TM) i7-3632 QM CPU @ 2.50 GHz 2.50 GHz; 16.00 GB memory; and Windows 10 64-bit Operating System.

\section{SPARQL query statements}

The SPARQL statements to generate the miRNA and $\mathrm{MeSH}$ term lists in the two search boxes are as follows, where the PHP variable \$type is used to determine whether the client is requesting a miRNA or MeSH term, and the PHP variable \$input contains either a partial or exact miRNA or MeSH term. Note that each line of the query statement has a detailed explanation right above it (the line starting with a pound sign "\#”).

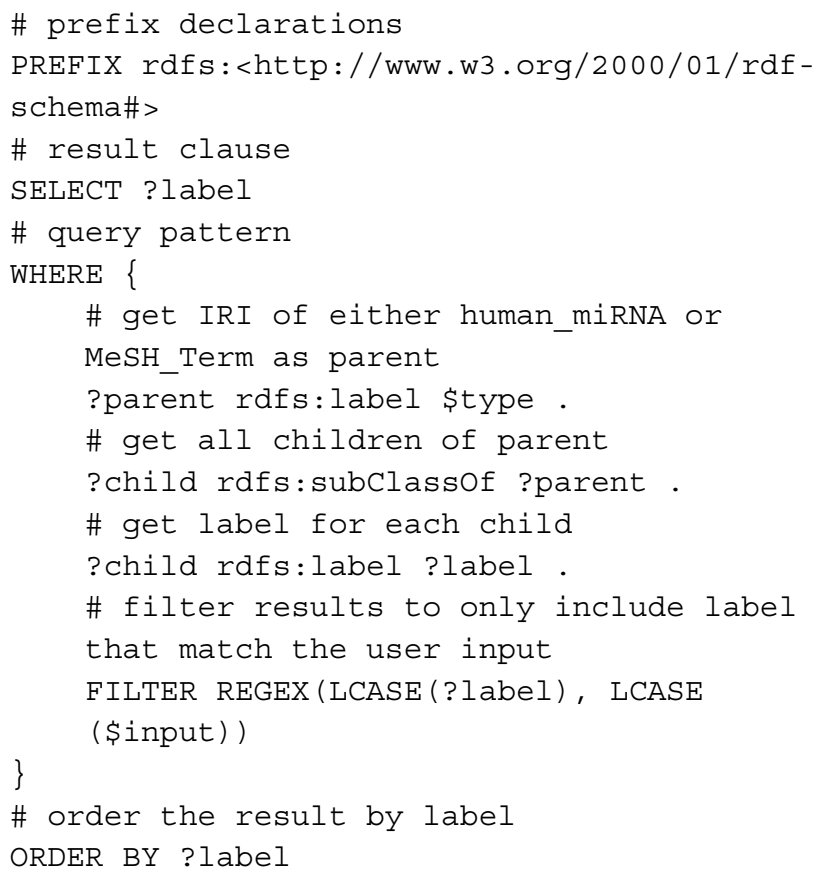

Suppose that the question of interest is: "What is the role of hsa-miR-125b-5p in cancer drug resistance?" The SPARQL statements are as follows. Similarly, all query statements have a detailed explanation.

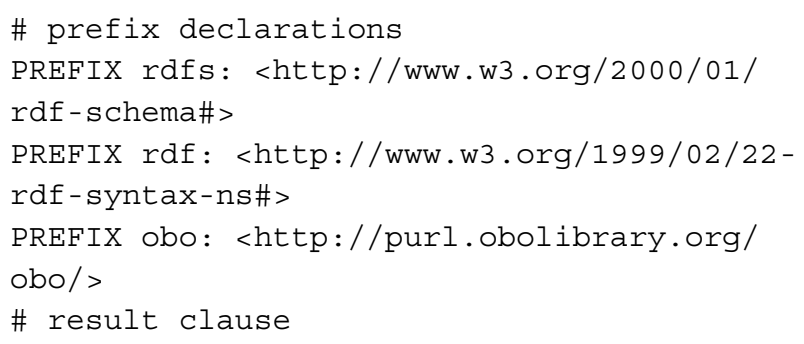

SELECT ?gene_symbol

\# group the gene ids together

(GROUP_CONCAT(DISTINCT ?g_id;

SEPARATOR=",") AS ?gene_id)

\# assign mdb_score to mirdb_score if

bound, otherwise assign 0

(MAX (COALESCE ( (?mdb_score),

?mab_score, 0)) AS ?mirdb_score)

\# assign ts_score to targetscan_score

if bound, otherwise assign 0

(MAX (COALESCE (?ts_score), ?ts_score, 0))

AS ?targetscan_score)

\# assign absolute value of mrnd_score

to miranda_score if bound, otherwise

assign 0

(MAX (COALESCE (ABS (?mrnd_score), 0)) AS

?miranda_score)

\# concatenate and group all pubmed ids

together, separated by a comma

(GROUP_CONCAT (DISTINCT ?pmid;

SEPARATOR=",") AS ?pubmed_ids)

\# query pattern

WHERE \{

\# get microRNA IRI with label

"hsa-miR-125b-5p"

?mirna rdfs:label "hsa-miR-125b-5p" .

\# get prediction that has_human_miRNA

micrORNA IRI

?prediction obo:OMIT_0000159 ?mirna.

\# get target where prediction has

miRNA_target_gene

?prediction obo:OMIT_0000160 ?target.

\# get gene symbol label of target

?target rdfs:label ?gene_symbol.

\# get target gene_id as g_id

?target obo:OMIT_0000109 ?g_id.

OPTIONAL \{

\# get prediction of type

prediction_from_Targetscan

?prediction rdf:type obo:

OMIT_0000019.

\# get prediction score as ts_score ?prediction obo:OMIT_0000108

?ts_score

\} .

OPTIONAL \{

\# get prediction of type

prediction_from_miRDB

?prediction rdf:type obo:OMIT_ 0000020 .

\# get prediction score as mdb_score ?prediction obo:OMIT_0000108 ?mdb_ score

\} . 


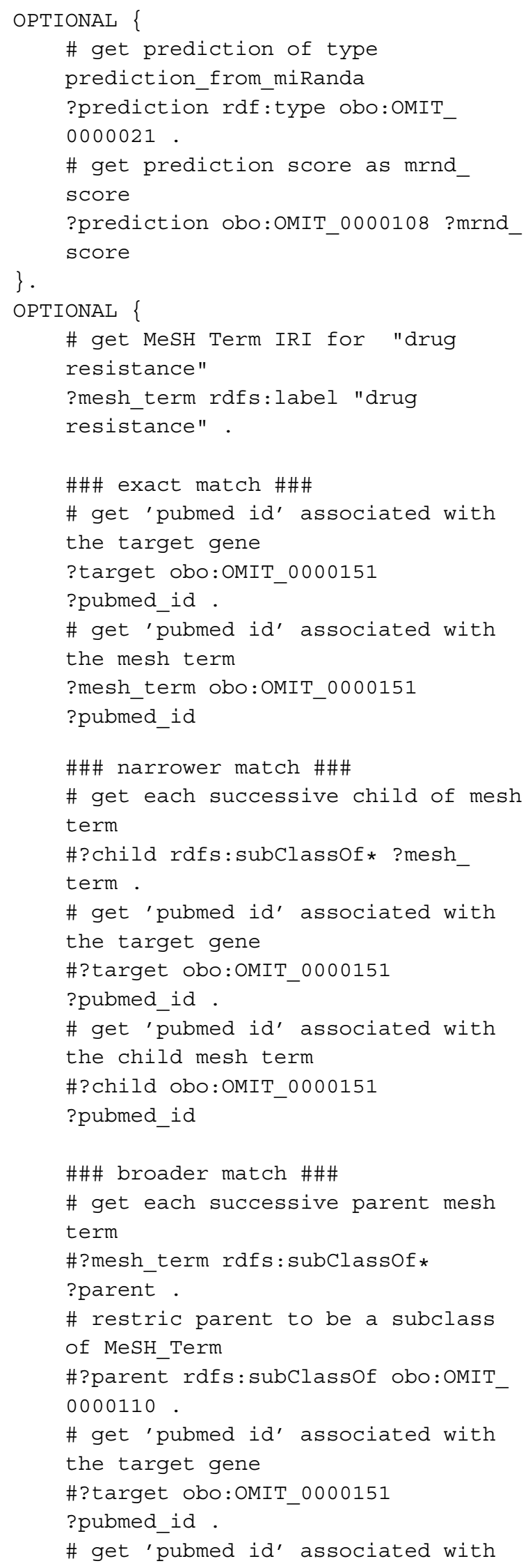

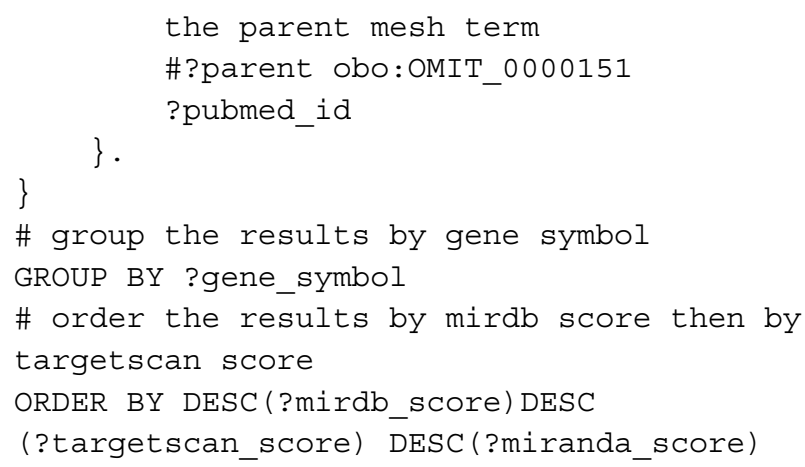

\section{Search results and discussion}

Corresponding to the aforementioned question of interest, Fig. 5 demonstrates the search results from a query on hsa-miR-125b-5p along with a MeSH-term filter "drug resistance".

- The "Candidate Targets" column contains all targets predicted by at least one target prediction database. The user can choose a prediction database and sort all targets by the scores, in descending order, from the selected database.

- The "Predicted By" column shows that each target is predicted by which database(s), along with the Web link(s) to these database(s).

- The "Publications" column links to all PubMed publications that are relevant to the search and filtering criteria. In this example, the criteria for any line are: the predicted target on that line, the miRNA hsa-miR-125b-5p, and the MeSH-term filter "drug resistance."

- The "GO Annotations" column connects to GO annotation results of each predicted target and the miRNA hsa-miR-125b-5p, respectively.

- Pathway analysis through DAVID can be performed on selected targets, either using the checkboxes to the left of the table or clicking the "Select All Targets" checkbox. Additionally, the user can select the desired tool to perform such analysis, "Gene Functional Classification," "Functional Annotation Clustering," "Functional Annotation Summary," and so forth.

- The whole result table can be downloaded in two different formats (tab-delimited text or CSV format); the user is also able to download only the predicted targets (selected ones or all).

We examined the search results demonstrated in this example, and our observations are summarized below.

1. Effective querying and accurate search results.

- Potential targets from all three miRNA target prediction databases (miRDB, TargetScan, and 


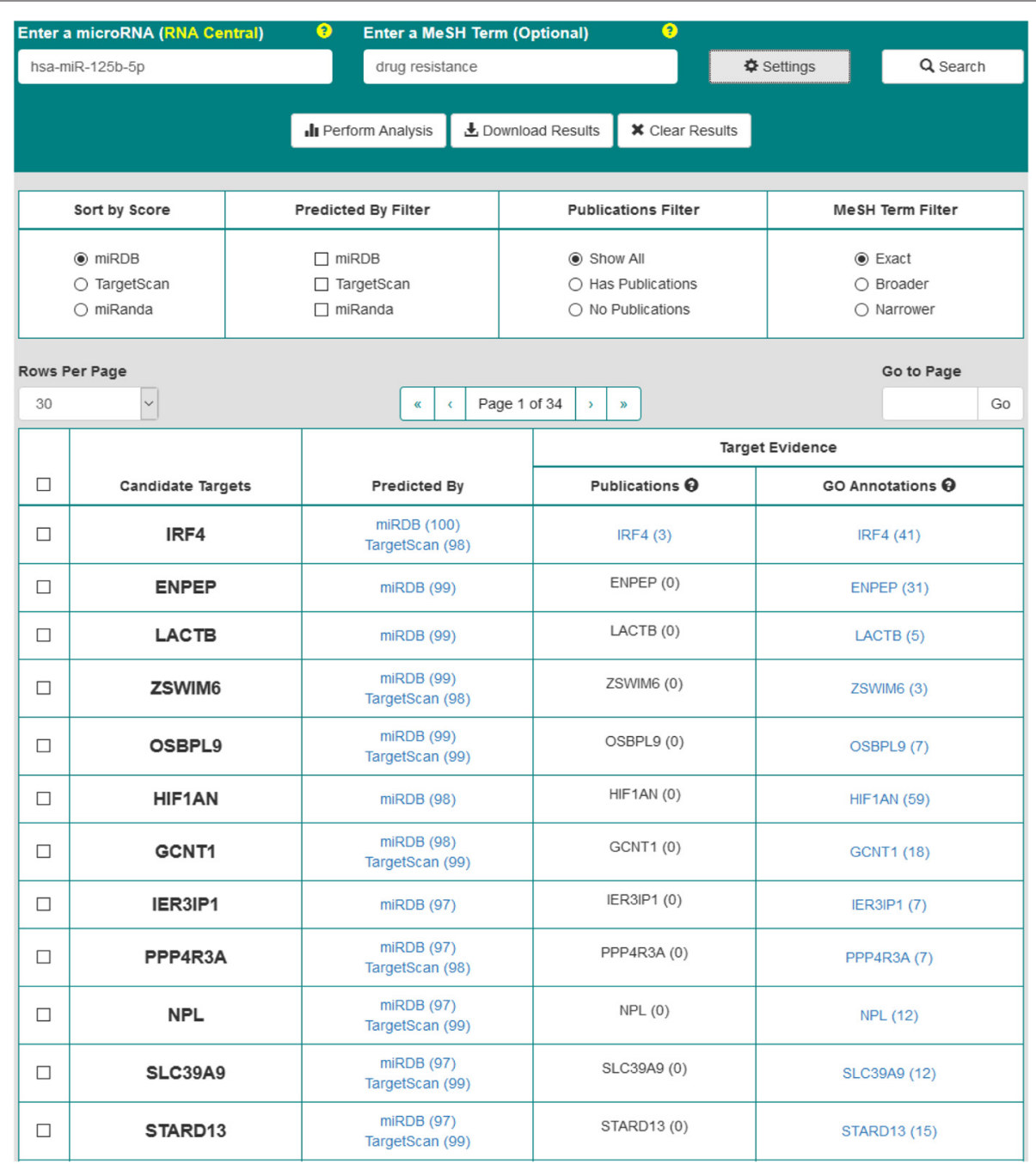

Fig. 5 Search results for the question of "What is the role of hsa-miR-125b-5p in cancer drug resistance?"

miRanda) were correctly retrieved. There were 476 and 924 targets from miRDB and

TargetScan, respectively; and there were 323 common targets. Consequently, a total of 1077 distinct targets were retrieved in the table when the "Predicted by Any Database" filter was chosen. Note that the miRanda database did not contain prediction results for the miRNA hsa-miR-125b-5p; therefore, no results appeared in the table when the display filter was set to "Predicted by All Databases." In fact, this observation further verified the effectiveness of the OmniSearch system.

- Relevant papers according to the search criteria were successfully retrieved. For example, two publications (PMID: 2497002 and 22808086) were retrieved for the predicted target LIN28A, supporting the conclusion that "Lin28A contributes to cancer drug resistance;" and three publications (PMID: 21823019, 24643683, and 19463775) were retrieved for the predicted target BAK1, supporting another conclusion that "BAK1 has an important role in cancer drug response and drug resistance."

- RNA Central annotations and GO annotations were correctly obtained. In this example query, a total of five sequences regarding the miRNA hsa-miR-125b-5p were retrieved from RNA Central annotations, and GO annotations for all predicted targets were retrieved as well. For example, a total of $117 \mathrm{GO}$ annotations (GO_REF:0000038, GO_REF:0000033, and so forth) were retrieved regarding a potential target, BAK1.

- Based on the above knowledge returned in the OmniSearch GUI, regarding the example 
question of "What is the role of hsa-miR-125b-5p in cancer drug resistance?" end users obtained the following answer: It is reasonable to speculate that expression of the miRNA hsa-miR-125b-5p contributes to cancer drug resistance, possibly through its suppression of expression for target genes BAK1 and/or LIN28A.

Discussion:

(1) miRDB, TargetScan, and miRanda databases have quite different meanings among each other in terms of their database entities. Due to the underlying OMIT and the formally defined semantics in the ontology, the OmniSearch system was able to effectively integrate the prediction results from all three databases. Note that conventional, database-oriented techniques can also implement such integration; however, inflexible, ad-hoc hard-coding will be required.

(2) To retrieve a correct set of relevant papers requires accessing numerous heterogeneous data sources such as NCBI Gene, PubMed, HGNC, and $\mathrm{MeSH}$. Without the common data elements defined in the OMIT and the thereafter semantic technologies including semantic annotation and data integration, it would have been extremely challenging to effectively integrate data from these sources, which is the case in database-oriented search and querying.

(3) As discussed earlier in "OMIT reconstruction" Section, the OMIT is closely connected with the GO by importing a set of GO terms. Compared with data integration based on traditional, relational databases, our approach has further facilitated integrating data about GO annotations.

2. More efficient querying process.

- One-stop visit rather than accessing different data sources separately, resulting in about $60 \%$ of time saved for end users.

- DAVID analysis was performed in a more efficient manner due to the target gene list automatically generated by the system. resulting in about $50 \%$ of time saved for end users.

- It was easier to compare different prediction results among miRDB, TargetScan, and miRanda databases, resulting in about $60 \%$ of time saved for end users.

- The above percentages of saved time were calculated as follows: We asked the aforementioned domain experts to perform a given set of queries using their conventional methods; next, they performed the same set of queries through the OmniSearch GUI; and finally, the saved time for all domain experts were averaged. Greater details on the system time and saved time for end users are contained in Table 4.

- Applying the MeSH-term filter resulted in a much smaller number of relevant publications returned. For example, 50 vs. 16 for the target $\mathrm{ABCC} 5,13$ vs. 2 for the target $\mathrm{DPH} 2$, and 31 vs. 3 for the target FOXQ1. More examples are demonstrated in Table 5.

\section{Discussion:}

(1) The reduced time spent by users was due to both data integration and the more accurate semantics defined in the ontology.

(2) In an non-ontology software system, to filtering on $\mathrm{MeSH}$ terms almost unavoidably results in hard-coding some ad-hoc searching rules in source code. On the contrary, semantics-oriented systems, such as OmniSearch, can well handle this issue in a more efficient manner. By decoupling domain knowledge from source code, ontologies and software applications can be developed independently, leading to more flexible software development.

(3) Based on the is_a relation, the OmniSearch system can perform logic reasoning over the ontology concept hierarchy (that is, both broader and narrower terms of the ontology term of interest), thus greatly improving the flexibility of search and query capability. For example, after a MeSH term is chosen by users, they are able to search the exact $\mathrm{MeSH}$ term, or its broader terms (i.e., ancestor terms) and narrower terms (i.e., offspring terms) defined in the ontology. Such results would not have been obtained without semantic technologies because systems based on relational databases are not able to perform any logical reasoning. Of course, users can still manually perform numerous queries and then obtain similar results as obtained from our system. However, such manual querying is significantly more time-consuming and labor-intensive, and more importantly, error-prone. (4) Cross-referencing among miRDB, TargetScan, and miRanda prediction results was made much easier because relevant database entities have already been formally defined in the OMIT. In other words, unambiguous semantics was accurately encoded with common data elements provided by the ontology, resulting in successful data sharing and exchanging among heterogeneous data sources.

(5) We asked the aforementioned domain experts to verify the accuracy of MeSH-term filtering. Because all returned publications contained the corresponding $\mathrm{MeSH}$ term, the Precision measure 
Table 4 The system time and saved time for end users

\begin{tabular}{|c|c|c|c|c|c|c|c|}
\hline Query & $\begin{array}{l}\text { First search } \\
\text { criterion }\end{array}$ & $\begin{array}{l}\text { Second search } \\
\text { criterion }\end{array}$ & $\begin{array}{l}\text { System time } \\
\text { (seconds) }\end{array}$ & $\begin{array}{l}\text { User time } \\
\text { (seconds) }\end{array}$ & $\begin{array}{l}\text { Percentage of saved } \\
\text { time for end users }\end{array}$ & $\begin{array}{l}\text { Percentage of saved } \\
\text { time on DAVID analysis }\end{array}$ & $\begin{array}{l}\text { Percentage of saved } \\
\text { time on result comparison }\end{array}$ \\
\hline 1 & hsa-miR-1231 & cell movement & 2.51 & 10 & $62 \%$ & $55 \%$ & $61 \%$ \\
\hline 2 & hsa-miR-1288-5p & cell proliferation & 2.89 & 9 & $61 \%$ & $51 \%$ & $62 \%$ \\
\hline 3 & hsa-miR-143-3p & mitosis & 5.54 & 10 & $61 \%$ & $52 \%$ & $60 \%$ \\
\hline 4 & hsa-miR-192-5p & leukemic infiltration & 2.24 & 8 & $53 \%$ & $53 \%$ & $59 \%$ \\
\hline 5 & hsa-miR-216a-5p & $\begin{array}{l}\text { drug resistance, } \\
\text { multiple }\end{array}$ & 4.09 & 11 & $65 \%$ & $55 \%$ & $62 \%$ \\
\hline 6 & hsa-miR-29c-3p & recurrence & 8.99 & 11 & $68 \%$ & $53 \%$ & $63 \%$ \\
\hline 7 & hsa-miR-3155a & dna cleavage & 1.21 & 6 & $53 \%$ & $47 \%$ & $55 \%$ \\
\hline 8 & hsa-miR-320b & drug resistance & 17.59 & 18 & $73 \%$ & $51 \%$ & $66 \%$ \\
\hline 9 & hsa-miR-3622a-5p & entosis & 0.30 & 6 & $51 \%$ & $43 \%$ & $57 \%$ \\
\hline 10 & hsa-miR-371b-5p & $\begin{array}{l}\text { mitochondrial } \\
\text { dynamics }\end{array}$ & 3.89 & 12 & $66 \%$ & $59 \%$ & $64 \%$ \\
\hline 11 & hsa-miR-3934-5p & dna methylation & 0.93 & 8 & $61 \%$ & $45 \%$ & $59 \%$ \\
\hline 12 & hsa-miR-4263 & mutagenesis & 1.65 & 6 & $52 \%$ & $46 \%$ & $56 \%$ \\
\hline 13 & hsa-miR-4431 & $\begin{array}{l}\text { mitochondrial } \\
\text { degradation }\end{array}$ & 0.17 & 6 & $53 \%$ & $47 \%$ & $55 \%$ \\
\hline 14 & hsa-miR-4505 & $\begin{array}{l}\text { cell transformation, } \\
\text { neoplastic }\end{array}$ & 4.25 & 10 & $63 \%$ & $55 \%$ & $61 \%$ \\
\hline 15 & hsa-miR-4648 & cell polarity & 0.71 & 6 & $52 \%$ & $45 \%$ & $57 \%$ \\
\hline 16 & hsa-miR-4700-3p & $\begin{array}{l}\text { neoplasm regression, } \\
\text { spontaneous }\end{array}$ & 1.56 & 7 & $53 \%$ & $51 \%$ & $59 \%$ \\
\hline 17 & hsa-miR-4756-5p & endocytosis & 3.76 & 10 & $67 \%$ & $53 \%$ & $62 \%$ \\
\hline 18 & hsa-miR-4802-3p & $\begin{array}{l}\text { drug resistance, } \\
\text { microbial }\end{array}$ & 1.67 & 7 & $55 \%$ & $47 \%$ & $59 \%$ \\
\hline 19 & hsa-miR-501-3p & insulin resistance & 1.78 & 8 & $57 \%$ & $43 \%$ & $61 \%$ \\
\hline 20 & hsa-miR-520a-3p & ubiquitination & 13.31 & 17 & $75 \%$ & $55 \%$ & $65 \%$ \\
\hline Average & - - - - & -——-— & 3.95 & 9.30 & $60.05 \%$ & $50.30 \%$ & $60.15 \%$ \\
\hline
\end{tabular}

was evaluated as $100 \%$. As for the Recall measure, it took a much longer time to evaluate because we needed to identify all publications that were incorrectly filtered out by the system. For example, there were three (one, resp.) publications relevant to CSNK2A1 (DVL3, resp.) that should not have been filtered out. More such examples are demonstrated in Table 6. Overall, an average Recall of $73 \%$ was achieved, meaning that while a user is able to obtain desired knowledge in a much more efficient manner (by reading significantly less publications, as shown in Table 5), the potential information lost is rather low.

3. Friendly user interface.

- For both search boxes, a list of partially matching terms were presented in a drop-down box as users typed in the box. Users were also allowed to not to type in anything, in which case all terms will be presented.

- The "Rows Per Page" drop-down and pagination control helped users to easily navigate among all predicted targets.

- A set of display filters were designed to allow users to conveniently and freely customize their preferred way to view retrieved results from various facets. For example, results can be sorted by the prediction score from any selected prediction database; users can choose to view only results that have publication evidence, or does not have such evidence, or both; and so forth.

- Flexible download options were provided, and all downloaded documents had self-explanatory, meaningful file names that contain the search 
Table 5 Reduced number of publications after applying the MeSH-term filter "drug resistance"

\begin{tabular}{|c|c|c|c|}
\hline $\begin{array}{l}\text { Target gene } \\
\text { symbol }\end{array}$ & $\begin{array}{l}\text { Original number } \\
\text { of papers }\end{array}$ & $\begin{array}{l}\text { Number of papers } \\
\text { after MeSH filtering }\end{array}$ & $\begin{array}{l}\text { Percentage } \\
\text { reduced }\end{array}$ \\
\hline $\mathrm{ABCC} 5$ & 50 & 16 & $68 \%$ \\
\hline DPH2 & 13 & 2 & $85 \%$ \\
\hline FOXQ1 & 31 & 3 & $90 \%$ \\
\hline CIAPIN1 & 43 & 4 & $91 \%$ \\
\hline SLC38A9 & 12 & 1 & $92 \%$ \\
\hline MCL1 & 452 & 31 & $93 \%$ \\
\hline MKNK2 & 30 & 2 & $93 \%$ \\
\hline BAG4 & 32 & 2 & $94 \%$ \\
\hline ARID3B & 18 & 1 & $94 \%$ \\
\hline HSPB2 & 79 & 4 & $95 \%$ \\
\hline THEMIS2 & 20 & 1 & $95 \%$ \\
\hline BAK1 & 266 & 11 & $96 \%$ \\
\hline SULT4A1 & 27 & 1 & $96 \%$ \\
\hline FUT4 & 57 & 2 & $96 \%$ \\
\hline GPC6 & 29 & 1 & $97 \%$ \\
\hline DDX54 & 29 & 1 & $97 \%$ \\
\hline MBD1 & 58 & 2 & $97 \%$ \\
\hline PRDM1 & 118 & 4 & $97 \%$ \\
\hline DTNB & 30 & 1 & $97 \%$ \\
\hline LIN28A & 91 & 3 & $97 \%$ \\
\hline SIRT7 & 33 & 1 & $97 \%$ \\
\hline ZBTB7A & 67 & 2 & $97 \%$ \\
\hline NCOR2 & 240 & 7 & $97 \%$ \\
\hline ТTPA & 35 & 1 & $97 \%$ \\
\hline MAP3K10 & 35 & 1 & $97 \%$ \\
\hline SGPL1 & 36 & 1 & $97 \%$ \\
\hline MYO18A & 36 & 1 & $97 \%$ \\
\hline EIF4EBP1 & 217 & 6 & $97 \%$ \\
\hline LIMK1 & 109 & 3 & $97 \%$ \\
\hline TP53INP1 & 37 & 1 & $97 \%$ \\
\hline CYTH1 & 39 & 1 & $97 \%$ \\
\hline SLC7A1 & 41 & 1 & $98 \%$ \\
\hline
\end{tabular}

date, "Query_Results_for_hsa-miR-125b-5p2015-12-05.csv" and

“Target_List_for_hsa-miR-125b-5p-2015-12-

05.txt" for example.

\section{Conclusions}

As a special class of ncRNAs, miRNAs have been demonstrated to play important roles in various biological and pathological processes. Because miRNAs realize their functions by regulating respective targets, it is critical to
Table 6 An example set of publications correctly/incorrectly filtered by "drug resistance"

\begin{tabular}{|c|c|c|c|}
\hline Gene symbol & $\begin{array}{l}\text { Total number of } \\
\text { publications } \\
\text { without applying } \\
\text { the "drug resistance" } \\
\text { filter }\end{array}$ & $\begin{array}{l}\text { Total number } \\
\text { of publications } \\
\text { that contain the } \\
\text { " MeSH term "drug } \\
\text { resistance" }\end{array}$ & $\begin{array}{l}\text { Total number of } \\
\text { incorrectly filtered } \\
\text { publications }\end{array}$ \\
\hline IRF4 & 130 & 3 & 0 \\
\hline ARID3B & 18 & 1 & 0 \\
\hline SGPL1 & 36 & 1 & 0 \\
\hline ESRRA & 131 & 3 & 0 \\
\hline PAFAH1B1 & 129 & 1 & 0 \\
\hline ETS1 & 287 & 5 & 0 \\
\hline TTPA & 35 & 1 & 0 \\
\hline DVL3 & 60 & 1 & 1 \\
\hline THEMIS2 & 20 & 1 & 0 \\
\hline VTCN1 & 66 & 1 & 0 \\
\hline WDR5 & 128 & 1 & 0 \\
\hline ETV6 & 198 & 4 & 0 \\
\hline TAZ & 74 & 1 & 0 \\
\hline IL6R & 300 & 1 & 0 \\
\hline $\mathrm{DPH} 2$ & 13 & 2 & 0 \\
\hline BTG2 & 84 & 1 & 0 \\
\hline CYP24A1 & 146 & 2 & 0 \\
\hline LIN28A & 91 & 3 & 0 \\
\hline TRPS1 & 69 & 1 & 0 \\
\hline CSNK2A1 & 619 & 5 & 3 \\
\hline TP53INP1 & 37 & 1 & 0 \\
\hline GPC6 & 29 & 1 & 0 \\
\hline DICER1 & 291 & 3 & 0 \\
\hline
\end{tabular}

identify and analyze miRNA-target interaction data to better explore and delineate miRNA functions. Semantic technologies and domain ontologies have been utilized to overcome limitations of conventional miRNA knowledge acquisition methods. To this end, we followed the research direction identified in our previous investigations regarding the establishment of common data elements and data exchange standards in the miRNA research. Specifically, our major scientific contributions in this paper are:

- We have significantly improved the OMIT ontology by: (1) following a modularized ontology design; (2) encoding all 1884 human miRNAs; and (3) setting up a GitHub project site along with an issue tracker for more effective community collaboration on the ontology development. The up-to-date ontology file is accessible at: http://purl.obolibrary.org/obo/omit.owl.

- Based upon the OMIT, we built the OmniSearch semantic search system, accessible at: http:// 
omnisearch.soc.southalabama.edu/index.php/ Software. Our experimental results demonstrated promising performance of OmniSearch. Consequently, more effective, more efficient miRNA-related knowledge capture has been achieved.

Finally, some research directions are envisioned as follows for our future work.

(1) To investigate a new set of filters to perform a wider scope of ontology reasoning. For example, potential filters can be developed according to different miRNA categories such as: oncogenic or tumor-suppressive miRNAs; individual tissues and/or cell lines in which miRNAs are expressed; and the gene family group to which miRNAs belong.

(2) To verify the consistency of contents retrieved from different data resources is another important future research topic. It is not trivial to resolve conflicting facts among different sources.

(3) It would be terrific for users to have more flexible options in further exploiting the semantics of the domain. Note that to construct more flexible queries will involve natural language processing (NLP) techniques, which are beyond the scope of this paper. Nevertheless, such an interesting topic can be considered in the future.

\section{Endnote}

${ }^{1}$ There are 103 and 18 OMIT-specific terms and relations, respectively.

\section{Competing interests}

The authors declare that they have no competing interests.

\section{Authors' contributions}

All authors performed requirements analysis. HJS contributed to GUI development. JH, AR, YL, HJS, and FG contributed to ontology development, term definition, and annotation examples. All authors read and approved the final manuscript.

\section{Acknowledgements}

Funding for Huang, I was provided in part by the National Cancer Institute $(\mathrm{NCl})$ at the National Institutes of Health $(\mathrm{NIH})$, under the Award Number U01CA180982. Funding for Borchert, GM was provided in part by Natural Science Foundation (NSF) CAREER grant 1350064 (GMB) awarded by Division of Molecular and Cellular Biosciences (with co-funding provided by the NSF EPSCoR program) and in part by the Abraham A. Mitchell Cancer Research Fund. The views contained in this paper are solely the responsibility of the authors and do not represent the official views, either expressed or implied, of the NIH, NSF, the U.S. Government, or the Abraham A. Mitchell Cancer Research Fund.

\footnotetext{
Author details

${ }^{1}$ School of Computing, University of South Alabama, Mobile, Alabama 36688-0002, USA. ${ }^{2}$ Computer and Information Science Department, University of Oregon, Eugene, Oregon 97403-1202, USA. ${ }^{3}$ Miracle Query, Inc., Eugene, Oregon 97403-1202, USA. ${ }^{4}$ Department of Philosophy, University at Buffalo, Buffalo, New York 14260-4150, USA. ${ }^{5}$ Genome Informatics, The Jackson Laboratory, Bar Harbor, Maine 04609-1523, USA. ${ }^{6}$ Department of Biomedical Informatics, University of Utah, Salt Lake City, Utah 84112-5775, USA. ${ }^{7}$ Department of Biochemistry and Molecular and Cellular Biology, Georgetown University Medical Center, Washington D.C. 20007-1485, USA. ${ }^{8}$ Center for
}

Computational Science, University of Miami, Miami, Florida 33146-2960, U.S.A. ${ }^{9}$ Department of Microbiology and Immunology, First Affiliated Hospital, Kunming Medical University, Kunming, Yunnan 650032, China. ${ }^{10}$ Department of Radiation Oncology, Washington University School of Medicine, St. Louis, Missouri 63110-0001, USA. ${ }^{11}$ Mitchell Cancer Institute, University of South Alabama, Mobile, Alabama 36604-1405, USA. ${ }^{12}$ Department of Biology, University of South Alabama, Mobile, Alabama 36688-0002, USA. ${ }^{13}$ School of Dental Medicine, University at Buffalo, Buffalo, New York 14214-8006, USA.

Received: 15 December 2015 Accepted: 12 April 2016

Published online: 10 May 2016

\section{References}

1. Zhao YH, Zhou M, Liu H, Khong HT, Yu DH, Fodstad O, Tan M. Upregulation of lactate dehydrogenase-A by ErbB2 through heat shock factor 1 promotes breast cancer cell glycolysis and growth. Oncogene. 2009;28(42):3689-701.

2. Liu Z, Liu H, Desai S, Schmitt D, Zhou M, Khong HT, Klos KS, McClellan S, Fodstad O, Tan M. MiR-125b functions as a key mediator for snail-induced stem cell propagation and chemoresistance. J Biol Chem. 2013;288(6):4334-4345.

3. Lu Z. PubMed and beyond: a survey of web tools for searching biomedical literature. Database. 2011;2011:1-13.

4. miRDB . [Online]. Available: http://mirdb.org/miRDB/. Accessed 19 Mar 2016.

5. TargetScan. [Online]. Available: http://www.targetscan.org. Accessed 19 Mar 2016.

6. miRanda. [Online]. Available: http://www.microrna.org. Accessed 19 Mar 2016.

7. DAVID Bioinformatics Resources. [Online]. Available: https://david.ncifcrf. gov/home.jsp. Accessed 19 Mar 2016.

8. NCBI Gene. [Online]. Available: http://ncbi.nlm.nih.gov/gene. Accessed 19 Mar 2016

9. Medical Subject Headings Database. [Online]. Available: https://www. nlm.nih.gov/mesh/. Accessed 19 Mar 2016

10. HUGO Gene Nomenclature Committee (HGNC) Database. [Online]. Available: http://www.genenames.org/. Accessed 19 Mar 2016.

11. NCBI Nucleotide. [Online]. Available: ncbi.nlm.nih.gov/nucleotide/.

12. Huang J, Tan M, Dou D, He L, Townsend C, Rudnick R, Hayes P. MiRNA Ontology for Target Prediction in Human Cancer. In: Proc. 1st ACM International Conference on Bioinformatics and Computational Biology, ACM-BCB-2010. Niagara Falls, NY: ACM Press; 2010.

13. Townsend C, Huang J, Dou D, Dalvi S, Hayes P, He L, Lin W, Liu H, Rudnick R, Shah H, Sun H, Wang X, Tan M. OMIT: Domain Ontology and Knowledge Acquisition in MicroRNA Target Prediction. In: Proc. 9th Intl' Conference on Ontologies, DataBases, and Applications of Semantics, ODBASE-2010. Crete, Greece: Springer-Verlag; 2010.

14. Huang J, Townsend C, Dou D, Liu H, Tan M. OMIT: a domain-specific knowledge base for MicroRNA target prediction. Pharm Res. 2011;28(12): $3101-4$

15. Huang J, Dang J, Lu X, Dou D, Blake J, Gerthoffer W, Tan M. An Ontology-Based MicroRNA Knowledge Sharing and Acquisition Framework. In: Proc. BHI Workshop at 2012 IEEE International Conference on Bioinformatics and Biomedicine, BIBM-2012. Philadelphia, PA: IEEE Computer Society Press; 2012.

16. Huang J, Dang J, Lu X, Xiong M, Gerthoffer W, Tan M. Semi-Automated microRNA Ontology Development based on Artificial Neural Networks. In: Proc. 2013 IEEE International Conference on Bioinformatics and Biomedicine, (BIBM-2013). Shanghai, China: IEEE Computer Society Press; 2013.

17. Huang J, Dang J, Borchert GM, Eilbeck K, Zhang H, Xiong M, Jiang W, Wu H, Blake JA, Natale DA, Tan M. OMIT: Dynamic, Semi-Automated Ontology Development for the microRNA Domain. PLOS ONE. 2014;9(7): $1-16$.

18. OBO Library. [Online]. Available: http://obofoundry.org. Accessed 19 Mar 2016.

19. NCBO BioPortal. [Online]. Available: https://bioportal.bioontology.org/. Accessed 19 Mar 2016.

20. Ashburner M, Ball C, Blake J, Botstein D, Butler H, Cherry J, Davis A, Dolinski K, Dwight S, Eppig J, Harris M, Hill D, Issel-Tarver L, Kasarskis A, Lewis S, Matese J, Richardson J, Ringwald M, Rubin G, Sherlock G. Gene 
ontology: tool for the unification of biology. The gene ontology consortium. Nat Genet. 2000;25(1):25-9.

21. Eilbeck K, Lewis $S$, Mungall $C$, Yandell M, Stein L, Durbin R, Ashburner M. The Sequence Ontology: a tool for the unification of genome annotations. Genome Biol. 2005;6(5).

22. Natale D, Arighi C, Barker W, Blake J, Bult C, Caudy M, Drabkin H, D'Eustachio P, Evsikov A, Huang H, Nchoutmboube J, Roberts N, Smith B, Zhang J, Wu C. The Protein Ontology: a structured representation of protein forms and complexes. Nucleic Acids Res. 2011;39:D539—45.

23. Hoehndorf R, Batchelor C, Bittner T, Dumontier M, Eilbeck K, Knight R, Mungall C, Richardson J, Stombaugh J, Westhof E, Zirbel C, Leontis N. The RNA Ontology (RNAO): An ontology for integrating RNA sequence and structure data. Appl Ontol. 2011;6(1):53-89.

24. Tran T, Cimiano P, Rudolph S, Studer R. Ontology-based interpretation of keywords for semantic search In: Aberer K, Choi K-S, Noy N, Allemang D, Lee K-I, Nixon L, Golbeck J, Mika P, Maynard D, Mizoguchi R, Schreiber G, Cudr-Mauroux P, editors. The Semantic Web. Berlin, Germany: Springer Berlin Heidelberg; 2007 vol. 4825. p. 523-36.

25. Premerlani WJ, Blaha MR. An approach for reverse engineering of relational databases. Commun ACM J. 1994;37(5):42-49, 134.

26. Stojanovic L, Stojanovic N, Volz R. Migrating data-intensive web sites into the Semantic Web. In: Proc. ACM symposium on Applied computing. Madrid, Spain: ACM Press; 2002. p. 1100-7.

27. Verheyden P, Bo JD, Meersman R. Semantically Unlocking Database Content Through Ontology-Based Mediation. Proc. SWDB 2004. Berlin, Germany: Springer-Verlag; 2004, pp. 109-26.

28. Lubyte L, Tessaris S. Extracting Ontologies from Relational Databases. Proc. Description Logics. Brixen-Bressanone, Italy: Free University of Bozen-Bolzano; 2007, pp. 122-6.

29. Chauhan R, Goudar R, Sharma R, Chauhan A. Domain ontology based semantic search for efficient information retrieval through automatic query expansion. In: Proc. Intelligent Systems and Signal Processing (ISSP), 2013 International Conference on. Vallabh Vidyanagar, Anand, India: IEEE Press; 2013. p. 397-402.

30. BFO. [Online]. Available: http://www.fomis.org/bfo/. Accessed 19 Mar 2016.

31. Smith B, Ceusters W, Klagges B, Köhler J, Kumar A, Lomax J, Mungall C, Neuhaus F, Rector A, Rosse C. Relations in biomedical ontologies. Genome Biol. 2005;6(5):1-15.

32. Huang J, Eilbeck K, Blake J, Dou D, Natale D, Ruttenberg A, Smith B, Zimmermann M, Jiang G, Lin Y, Wu B, He Y, Zhang S, Wang X, Zhang H, Liu Z, Tan M. A domain ontology for the non-coding rna field. In: Proc 2015 IEEE International Conference on Bioinformatics and Biomedicine, BIBM 2015. Washington: IEEE; 2015. p. 621-4.

33. OBI. [Online]. Available: http://obi-ontology.org/. Accessed 19 Mar 2016.

34. CHEBI. [Online]. Available: http://obofoundry.org/ontology/chebi.html. Accessed 19 Mar 2016.

35. IAO. [Online]. Available: http://obofoundry.org/ontology/iao.html. Accessed 19 Mar 2016.

36. Cell Line Ontology. [Online]. Available: http://obofoundry.org/ontology/ clo.html. Accessed 19 Mar 2016.

37. Uberon multi-species anatomy ontology. [Online]. Available: http:// obofoundry.org/ontology/uberon.html. Accessed 19 Mar 2016.

38. Human Disease Ontology. [Online]. Available: http://obofoundry.org/ ontology/doid.html. Accessed 19 Mar 2016.

39. The Apache Software Foundation. [Online]. Available: http://www. apache.org/. Accessed 19 Mar 2016.

40. PHP: Hypertext Preprocessor. [Online]. Available: http://php.net/. Accessed 19 Mar 2016.

41. Apache Jena Fuseki. [Online]. Available: http://jena.apache.org/ documentation/fuseki2/index.html. Accessed 19 Mar 2016.

42. miRBase. [Online]. Available: http://www.mirbase.org/. Accessed $19 \mathrm{Mar}$ 2016.

43. OMIT Project Site. [Online]. Available: http://omnisearch.soc. southalabama.edu. Accessed 19 Mar 2016

44. OMIT in OBO Library. [Online]. Available: http://www.obofoundry.org/cgibin/detail.cgi?id=omit. Accessed 19 Mar 2016.
45. OMIT in NCBO BioPortal. [Online]. Available: http://bioportal.bioontology. org/ontologies/OMIT. Accessed 19 Mar 2016.

46. OMIT Tracker. [Online]. Available: https://github.com/OmniSearch/OMITontology-files/issues. Accessed 19 Mar 2016.

47. RDF 1.1 Turtle: Terse RDF Triple Language. [Online]. Available: http:// www.w3.org/TR/turtle/. Accessed 19 Mar 2016.

\section{Submit your next manuscript to BioMed Central and we will help you at every step:}

- We accept pre-submission inquiries

- Our selector tool helps you to find the most relevant journal

- We provide round the clock customer support

- Convenient online submission

- Thorough peer review

- Inclusion in PubMed and all major indexing services

- Maximum visibility for your research

Submit your manuscript at www.biomedcentral.com/submit
Biomed Central 